\title{
A Case Report of COVID-Associated Catastrophic Antiphospholipid Syndrome Successfully Treated with Eculizumab
}

\author{
Anusha Chidharla (1D' \\ Salman B Syed (iD) ${ }^{2}$ \\ Tulika Chatterjee ${ }^{2}$ \\ Michael D Tarantino ${ }^{3}$ \\ 'Department of Medical Oncology, \\ Kansas University Cancer Center, \\ Kansas, KS, USA; ${ }^{2}$ Department of \\ Internal Medicine, University of Illinois \\ College of Medicine, Peoria, IL, USA; \\ ${ }^{3}$ Department of Hematology, Bleeding \\ and Clotting Disorders Institute, Peoria, \\ IL, USA
}

\begin{abstract}
Antiphospholipid syndrome (APS) is an autoimmune disorder characterized by multiple episodes of venous and arterial thromboses or recurrent fetal losses in the presence of antiphospholipid antibodies against $\beta_{2} \mathrm{GP} 1$, frequently accompanied by moderate thrombocytopenia. Catastrophic APS (CAPS) is a severe manifestation of APS. COVID-19 may have an intense hypercoagulable state in critically ill patients. SARS-CoV2 may potentiate pathogenic APS effects, including the activation of endothelial cells, monocytes, platelets, and complement, resulting in a proinflammatory state and prothrombotic events. The endothelial tropism of SARS-CoV2 may also modify the clinical presentation of COVID19 in susceptible individuals and trigger flares of underlying vascular diseases. We report a case of a 64-year-old woman with a history of triple-positive APS who had multiple thrombotic and bleeding episodes after being found to have a COVID-19 infection temporally associated with CAPS development that was successfully treated with eculizumab, preventing further macro- and microvascular thrombotic events at 1 month follow-up. Our case highlights the need for more research regarding the mechanism by which COVID-19 may potentiate APS and lead to the development of CAPS.
\end{abstract}

Keywords: antiphospholipid syndrome, phospholipid autoantibodies, catastrophic APS, COVID-19, severe acute respiratory syndrome coronavirus 2 , eculizumab

\section{Introduction}

Antiphospholipid syndrome (APS) is an autoimmune disorder characterized by multiple episodes of venous and arterial thromboses or recurrent fetal losses in the presence of antiphospholipid antibodies (aPL Abs) against $\beta_{2} \mathrm{GP} 1$, and is frequently accompanied by moderate thrombocytopenia. ${ }^{1}$ Rarely, APS may be associated with fulminant multiorgan failure or catastrophic APS (CAPS). CAPS is characterized by widespread small-vessel occlusions that lead to multiorgan failure. In an international study of CAPS-registry patients' lupus anticoagulant, IgG anticardiolipin and IgG anti $\beta_{2}$ GP Abs were the most often implicated aPL Abs in CAPS. Although affecting only $1 \%$ of patients with APS, CAPS is frequently fatal if not recognized and treated promptly. ${ }^{2}$ In spite of its clinical significance, with an approximate mortality rate of $40 \%$, the pathophysiology remains somewhat enigmatic. Activation of the complement is required for the full clinical manifestation of APS. APS serum activates the complement in vitro, and patients with CAPS have been found to have a high rate of complement-gene mutations. ${ }^{3}$ In
Correspondence: Anusha Chidharla 9219 West 157th Terrace, Overland Park, KS, 6622I, USA

Tel + I 913-568-5708

Email anushaaedma@gmail.com 
a descriptive analysis of 500 CAPS patients, the most common precipitating factor of CAPS was infection. ${ }^{4}$ Infections, including COVID-19, are considered trigger factors of CAPS. It could be possible that SARS-CoV2 can induce CAPS; however, no evidence has been found for this. A study of COVID-19 patients showed aPL Abs were common in critically ill patients. ${ }^{5}$ El Hasbani et al wrote a review article that discussed APL in the setting of COVID-19. ${ }^{6}$ Eculizumab inhibits the activation of complement $\mathrm{C} 5$, and is approved for use in paroxysmal nocturnal hemoglobinuria and atypical hemolytic uremic syndrome. We present a patient with a history of APS who had multiple thrombotic and bleeding episodes consistent with CAPS, likely triggered by SARS-CoV2 infection, that was successfully treated with eculizumab.

\section{Case Presentation}

A 64-year-old woman with a history of type 2 diabetes mellitus and triple-positive APS presented with acute encephalopathy. Her vital signs were normal on admission, with no significant physical examination findings except for altered mental status. Laboratory studies revealed hemoglobin $(\mathrm{Hb})$ concentration of $131 \mathrm{~g} / \mathrm{L}$, white blood cell (WBC) count of $11.8 \times 10^{9} / \mathrm{L}$, absolute lymphocyte count (ALC) of $1.5 \times 10^{9} / \mathrm{L}$, platelet count of $173 \times 10^{9} / \mathrm{L}$, and D-dimer of $0.54 \mu \mathrm{g} / \mathrm{mL}$. MRI of the brain revealed chronic microvascular ischemia. Therapeutic unfractionated heparin was initiated, given her history of APS and multiple venous thrombotic events. On day 10, she developed a fever and hypoxia and tested positive for SARS-Co V2. Dexamethasone and remdesivir were initiated. Remdesivir was discontinued after 3 days, due to worsening kidney function. Subsequent laboratory testing revealed $\mathrm{Hb}$ concentration $86 \mathrm{~g} / \mathrm{L}$, WBC count $11 \times 10^{9} / \mathrm{L}$, ALC $0.77 \times 10^{9} / \mathrm{L}$, and platelet count $93 \times 10^{9} / \mathrm{L}$. APL $\mathrm{Ab}$ testing revealed a positive lupus anticoagulant, cardiolipin IgG $>112 \mathrm{U} / \mathrm{mL}, \operatorname{IgA}>65 \mathrm{U} / \mathrm{mL}, \beta_{2} \mathrm{GP} 1 \mathrm{IgG}>112 \mathrm{U} / \mathrm{mL}$, and $\operatorname{IgA}>65 \mathrm{U} / \mathrm{mL}$. Her platelet count had decreased to $67 \times 10^{9} / \mathrm{L}$. A coagulation profile showed a prothrombin time of 23.3 seconds, international normalized ratio of 2.1, activated partial thromboplastin time of 132 seconds, fibrinogen activity of $832 \mathrm{mg} / \mathrm{dL}$, and D-dimer of $1.56 \mu \mathrm{g} /$ $\mathrm{mL}$. On the following day, the patient developed acute respiratory distress requiring noninvasive ventilation. CT of the chest demonstrated diffuse bilateral ground-glass opacity a $4.3 \mathrm{~cm}$ left adrenal mass, concerning in terms of adrenal hemorrhage (Figure 1). Intravenous (IV)

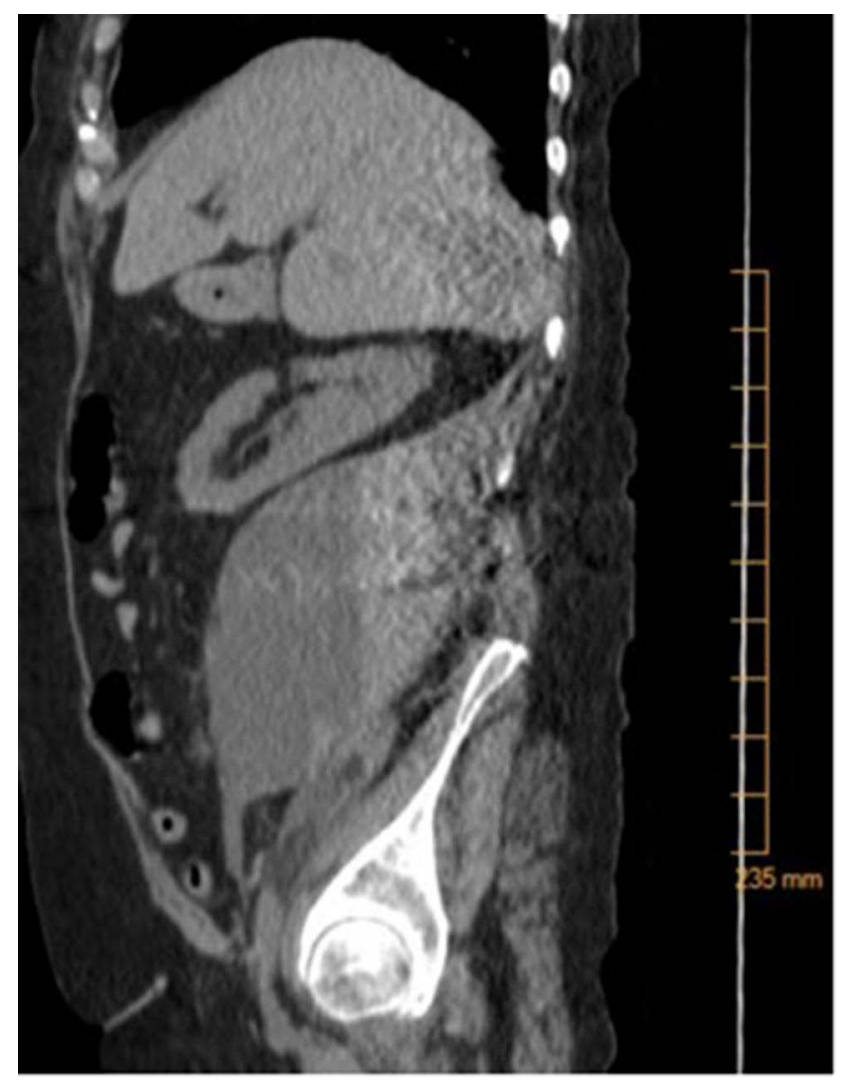

Figure I CT of abdomen and pelvis without contrast, showing large right-sided retroperitoneal mass $(10 \times 11 \times 16 \mathrm{~cm})$ concerning for hematoma of varying age.

methylprednisolone $500 \mathrm{mg}$ was initiated. On day 19 , the patient showed a significant decrease in $\mathrm{Hb}$ concentration and had retroperitoneal hemorrhage on imaging. Her anticoagulation was stopped. She later had acute venous thrombosis of the right superficial femoral vein. Because of intolerance to anticoagulation, an inferior vena cava filter was placed. She developed acute right-sided weakness on day 21 of admission, and the brain MRI showed new areas of subacute watershed infarctions with areas of concern in terms of hemorrhagic conversion (Figure 2). A direct antiglobulin test was positive, serum haptoglobin concentration was low, and serum lactate dehydrogenase concentration was elevated on further evaluation of anemia and thrombocytopenia, which raised concern regarding autoimmune hemolytic anemia. With new-onset ischemic stroke, acute venous thrombosis, and adrenal hemorrhage, the patient met the criteria for CAPS. She underwent single-volume plasma exchange daily for 5 consecutive days. She also received two doses of IV rituximab 1,000 $\mathrm{g}$ and two doses of intravenous immunoglobulin $1 \mathrm{mg} / \mathrm{kg}$. APL Abs and coagulation parameters were monitored every other day, and initially decreased, 


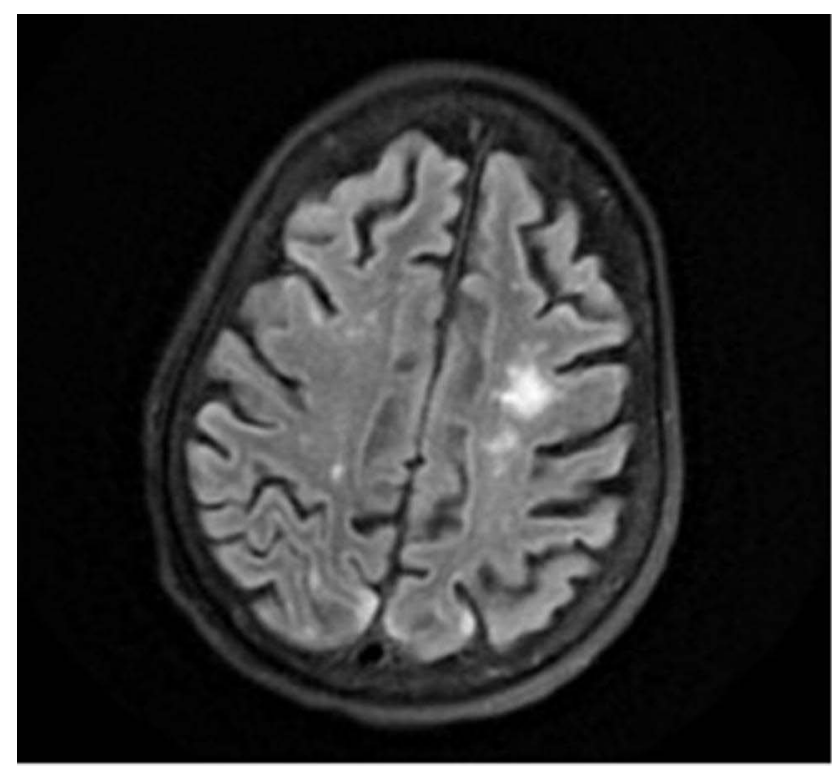

Figure 2 Brain MRI showing new areas representing subacute watershed infarctions with several punctate areas of acute infarction within the bilateral anterior cerebral artery/middle cerebral artery watershed territories.

but later continued to increase. Because of the rise in $\mathrm{Ab}$ titers and limited improvement in her clinical status, she was given IV eculizumab $900 \mathrm{mg}$ weekly for refractory CAPS. She received two doses of eculizumab prior to discharge, and was started on prophylactic enoxaparin. The patient did not have any recurrent thrombotic events, and her mentation and right-sided weakness had continued to improve at 1-month follow-up.

\section{Discussion}

CAPS is a severe manifestation of APS. It is characterized by multiple thromboses involving three or more organs, systems, or tissue types, histopathology of small-vessel occlusion in at least one organ, acuity of manifestations, and aPL Ab positivity. When all four criteria are present, a "definite CAPS" diagnosis is confirmed. With our patient, she met the criteria for CAPS. Known risk factors of CAPS include infection, surgery, malignancy, anticoagulation withdrawal, low INR, obstetric complications, drugs, and systemic lupus erythematosus flares. ${ }^{7}$

Critically ill patients with COVID-19 are known to have a profound hypercoagulable state. The exact mechanism of COVID-associated thrombophilia is still being elucidated, but it is likely to have a multifactorial etiopathogenesis. Hypoxia and prolonged immobilization are important predisposing factors, but the diffuse inflammatory state and resulting "cytokine storm" seem to be the primary triggers. ${ }^{6,8}$ In addition, endothelial dysfunction, characterized by increased levels of von Willebrand factor, systemic inflammation, activation of Toll-like receptors, a procoagulatory state, and tissue factor-pathway activation are among the suggested mechanisms. ${ }^{6}$ Another possible underrecognized mechanism may be the induction of aPL $\mathrm{Ab}$ production in severe COVID. ${ }^{9}$ Molecular mimicry leading to anti- $\beta_{2} \mathrm{GP} 1 \mathrm{Ab}$ production has also been a proposed cause of secondary APS and CAPS. A small case series reported elevated aPL Abs in COVID-19 patients, and CAPS is associated with consistently elevated aPL Abs. ${ }^{6,9-11}$

Given that mortality rate in CAPS is approximately $50 \%$, early identification and treatment is crucial. Nevertheless, due to the rarity of the disease, there have been no randomized controlled trials to guide therapy. The typical treatment approach for CAPS is multiagent therapy that includes high-dose corticosteroids, intravenous immunoglobulin, and plasma exchange. ${ }^{12}$ Recent reports suggest that rituximab treatment may improve clinical signs and immunological profiles in CAPS management, and it is thus used in patients who have failed to respond to the standard therapy described. ${ }^{13}$ In our patient's case, despite rituximab therapy, she continued to experience rising aPL $\mathrm{Ab}$ levels and worsening coagulation-profile results. As such, our patient received a trial of eculizumab, to which she responded well clinically. Also, the aPL titers and coagulation parameters decreased while our patient was on eculizumab. These parameters were consistently followed on an inpatient/outpatient basis.

Eculizumab is a humanized monoclonal $\mathrm{Ab}$ approved for use in paroxysmal nocturnal hemoglobinuria and atypical hemolytic uremic syndrome, but has been used in the treatment of refractory CAPS. ${ }^{14-16}$ To our knowledge, this is the first case describing successful treatment of COVIDassociated CAPS successfully treated with eculizumab. As complement activation plays a crucial role in animal models of APS thrombosis, eculizumab is considered a last resort for CAPS treatment. To date, data are restricted to eleven isolated case reports that indicated a dramatic improvement of CAPS after eculizumab. Inconsistent results with its use on clinical outcomes have been reported by Yelnik et al. ${ }^{17}$ Ongoing randomized trials are investigating the role of anti-C5 drugs in severe COVID19; however, several case series have suggested promising effects on clinical outcomes in patients with severe COVID-19. ${ }^{18}$ Ours is the first case describing successful treatment of COVID-associated CAPS with eculizumab. The absence of further episodes of thrombosis despite 
stagnant aPL Ab levels in our patient treated with eculizumab highlights the potential role of complementmediated thrombosis in CAPS.

\section{Conclusion}

In a subset of patients with severe COVID-19, increased production of aPL Abs may play a role in the development of CAPS. Studies are needed to evaluate the presence of aPL Abs and CAPS in patients with COVID-19. SARS-Co V2 may exaggerate the clinical manifestations of APS, including activation of endothelial cells, monocytes, platelets, and complement, resulting in proinflammatory and prothrombotic states. The endothelial tropism of SARSCoV2 may also modify the clinical presentation of COVID-19 in susceptible patients. Few studies have showen that patients with CAPS have mutations in complements, which increase complement activation. However, further studies are needed to elucidate the role of complement in CAPS. There have been case series reporting eculizumab use in refractory CAPS. Highquality studies like randomized control trials are needed to evaluate the efficacy of eculizumab in refractory CAPS. Our case highlights the need for more research regarding the mechanism(s) by which COVID-19 may potentiate APS predisposing to CAPS and treatment of refractory CAPS with eculizumab.

\section{Consent}

Written informed consent was obtained from the patient's health-care power of attorney/next of kin to have the case details and any accompanying results published. Institutional approval was not required to publish the case details.

\section{Disclosure}

Dr Michael D Tarantino is the CMO and CEO of the Bleeding and Clotting Disorders Institute, and reports grants and/or personal fees for consultancy, speakers' bureaux and/or clinical trial PI from Amgen, BioMarin, Dova, Genentech, Grifols, Octapharma, Principia, Sobi, Spark Therapeutics, Takeda, and UCB outside the submitted work. The authors report no other relevant conflicts of interest in this work.

\section{References}

1. Gezer S. Antiphospholipid syndrome. Dis Mon. 2003;49(12):696-741. doi:10.1016/j.disamonth.2003.10.001
2. Rodriguez-Pintó I, López-Benjume B, Espinosa G, Cervera R. Catastrophic antiphospholipid syndrome. Rev Colomb De Reumatol. 2021;28:39-43. doi:10.1016/j.rcreu.2021.02.004

3. Chaturvedi S, Braunstein EM, Yuan X, et al. Rare Germline mutations in complement regulatory genes make the antiphospholipid syndrome catastrophic. Blood. 2019;134(Supplement 1):4. doi:10.1182/blood-2019-129115

4. Rodríguez-Pintó I, Moitinho M, Santacreu I, et al. Catastrophic antiphospholipid syndrome (CAPS): descriptive analysis of 500 patients from the International CAPS registry. Autoimmun Rev. 2016;15 (12):1120-1124. doi:10.1016/j.autrev.2016.09.010

5. Xiao M, Zhang Y, Zhang S, et al. Antiphospholipid antibodies in critically ill patients with COVID-19. Arthritis Rheumatol. 2020;72 (12):1998-2004. doi:10.1002/art.41425

6. El Hasbani G, Taher AT, Jawad A, Uthman I. COVID-19, antiphospholipid antibodies, and catastrophic antiphospholipid syndrome: a possible association? Clin Med Insights Arthritis Musculoskelet Disord. 2020;13:1179544120978667. doi:10.1177/ 1179544120978667

7. Mendoza-Pinto C, García-Carrasco M, Cervera R. Role of infectious diseases in the antiphospholipid syndrome (including its catastrophic variant). Curr Rheumatol Rep. 2018;20(10):62. doi:10.1007/s11926018-0773-x

8. Merrill JT, Erkan D, Winakur J, James JA. Emerging evidence of a COVID-19 thrombotic syndrome has treatment implications. Nat Rev Rheumatol. 2020;16(10):581-589. doi:10.1038/s41584-0200474-5

9. Mendoza-Pinto C, Escárcega RO, García-Carrasco M, Bailey DJO, Gálvez-Romero JL, Cervera R. Viral infections and their relationship with catastrophic antiphospholipid syndrome: a possible pathogenic mechanism of severe COVID-19 thrombotic complications. J Intern Med. 2020;288(6):737-739. doi:10.1111/joim.13123

10. Upson S, Murphy M, Ashraf F, Shahzad S. Double trouble: a case of suspected antiphospholipid syndrome in the setting of hypercoagulable COVID-19 pneumonia. Chest. 2020;158(4):A862. doi:10.1016/j. chest.2020.08.801

11. Yin S, Huang M, Li D, Tang N. Difference of coagulation features between severe pneumonia induced by SARS-CoV2 and non-SARSCoV2. J Thromb Thrombolysis. 2020. doi:10.1007/s11239-02002105-8

12. Gansner JM, Berliner N. The rheumatology/hematology interface: CAPS and MAS diagnosis and management. Hematology. 2018;2018(1):313-317. doi:10.1182/asheducation-2018.1.313

13. Rymarz A, Niemczyk S. The complex treatment including rituximab in the management of catastrophic antiphospholid syndrome with renal involvement. BMC Nephrol. 2018;19(1):132. doi:10.1186/ s12882-018-0928-z

14. Kazzaz NM, McCune WJ, Knight JS. Treatment of catastrophic antiphospholipid syndrome. Curr Opin Rheumatol. 2016;28 (3):218-227. doi:10.1097/BOR.0000000000000269

15. Tinti MG, Carnevale V, Inglese M, et al. Eculizumab in refractory catastrophic antiphospholipid syndrome: a case report and systematic review of the literature. Clin Exp Med. 2019;19(3):281-288. doi:10.1007/s10238-019-00565-8

16. Nauseef JT, Lim HI, DeSancho MT. Successful outcome with eculizumab treatment in a patient with antiphospholipid syndrome presenting with an unusual thrombotic storm. J Thromb Thrombolysis. 2020. doi:10.1007/s11239-020-02343-w

17. Yelnik CM, Miranda S, Mékinian A, et al. Patients with refractory catastrophic antiphospholipid syndrome respond inconsistently to eculizumab. Blood. 2020;136(21):2473-2477. doi:10.1182/ blood.2020007499

18. Fodil S, Annane D. Complement inhibition and COVID-19: the story so far. ImmunoTargets Ther. 2021;10:273-284. doi:10.2147/ITT. S284830 


\section{Publish your work in this journal}

The Journal of Blood Medicine is an international, peer-reviewed, open access, online journal publishing laboratory, experimental and clinical aspects of all aspect pertaining to blood based medicine including but not limited to: Transfusion Medicine; Blood collection, Donor issues, Transmittable diseases, and Blood banking logistics; Immunohematology; Artificial and alternative blood based

Submit your manuscript here: http://www.dovepress.com/journal-of-blood-medicine-journal therapeutics; Hematology; Biotechnology/nanotechnology of blood related medicine; Legal aspects of blood medicine; Historical perspectives. The manuscript management system is completely online and includes a very quick and fair peer-review system. Visit http://www.dovepress.com/testimonials.php to read real quotes from published authors. 